\section{How-I-Do-lt}

Ann Liver Transplant 2021;1(2):140-145

https://doi.org/10.52604/alt.21.0023

Check for updates
ALT

pISSN 2765-5121

elSSN 2765-6098

\title{
Minimal-incision donor right hepatectomy for living donor liver transplantation
}

\author{
Tae-Yong Ha, Dong-Hwan Jung, Gi-Won Song, Gil-Chun Park, Shin Hwang
}

Division of Hepatobiliary Surgery and Liver Transplantation, Department of Surgery, Asan Medical Center, University of Ulsan College of Medicine, Seoul, Korea

Received October 5, 2021

Revised October 9, 2021

Accepted October 15, 2021

Published online October 22, 2021

Corresponding author: Shin Hwang Department of Surgery, Asan Medical Center, University of Ulsan College of Medicine, Olympic-ro 43-gil 88, Songpa-gu, Seoul 05505, Korea

E-mail: shwang@amc.seoul.kr https://orcid.org/0000-0002-9045-2531

(c) The Korean Liver Transplantation Society (c) (i) \& This is an Open Access article distributed under the terms of the Creative Commons Attribution Non-Commercial License (http://creativecommons.org/licenses/by-nc/4.0/) which permits unrestricted non-commercial use, distribution, and reproduction in any medium, provided the original work is properly cited.
For living donors, the second most important concern after the donor safety is the cosmetic aspect of abdominal incision. The present study aimed to present the technical details of minimal-incision laparotomy (MIL) in a case of living donor right hepatectomy with an eight minutes video clip. The recipient was a 57-year-old patient with alcoholic liver cirrhosis and the donor was his 28-year-old daughter of the recipient. The epigastrium area was narrow and the subcostal cartilages were elongated beyond the level of the umbilicus, so a 12-cm-long right subcostal incision was made. The right liver was mobilized with gentle traction. The right hepatic parenchyma was transected according to standard procedures and liver-hanging with a Penrose drain. A $780 \mathrm{~g}$-weighing right liver graft was harvested and pulled out through the skin incision. The liver graft was converted to a modified right liver graft with patch and conduit venoplasties of the outflow veins. Both the recipient and donor recovered uneventfully and have been doing well for 12 years after transplantation. We believe that MIL is a compromise option between conventional skin incision and total laparoscopic hepatectomy regarding cosmetics of the skin incision and donor safety, although it is a demanding procedure for donor surgeons.

Keywords: Living donor liver transplantation; Wound cosmetics; Donor safety; Wound scar; Minimal skin incision

\section{INTRODUCTION}

For living donors, the second most important concern after the donor safety is the cosmetic aspect of abdominal incision. During living donor hepatectomy, a partial liver graft should be pulled out intact through the skin incision, and therefore a sizable skin incision is inevitable $[1,2]$. So far, several shapes of skin incision have been performed for living donor hepatectomy to enhance wound cosmetics, including laparoscopic donor hepatectomy, video-assisted donor hepatectomy including hand-assisted laparoscopic surgery (HALS), and minimal skin incisions [1-8].

A mirrored L-incision has been the standard incision for living donor right hepatectomy in our institution because it provides a wide operative field regardless of the body dimension or the shape of donor subcostal cartilages. However, this incision has occasionally resulted in excessive wound scarring, which caused wound discomfort and complaints of cosmetic dissatisfaction in some donors. As a compromise option, we have performed donor right hepatectomy using a minimal-incision laparotomy (MIL). The present study aimed to present the technical details of MIL 
in donor right hepatectomy with an eight minutes video clip (Supplementary Video 1).

\section{CASE PRESENTATION}

The recipient was a 57-year-old patient with alcoholic liver cirrhosis (Fig. 1A). His body weight was $59 \mathrm{~kg}$ and the model for end-stage liver disease score was 14 . The liver function of the patient deteriorated progressively, therefore living donor liver transplantation (LDLT) was planned.

The living donor was a 28-year-old daughter of the recipient. Her body weight was $54 \mathrm{~kg}$ and her height was 168 $\mathrm{cm}$ (Fig. 1B). The epigastrium area was narrow and the subcostal cartilages were elongated beyond the level of the umbilicus, so a 12-cm-long right subcostal incision was made (Fig. 2A, B). Two subcostal retractors were gently applied to stretch the skin incision. Routine cholecystectomy was performed and a small tube was inserted to take an intraoperative cholangiogram. The cholangiogram showed normal bifurcation of the hilar bile duct.

The right liver was gently retracted toward the medio-ventral side, and then the liver was carefully dissected from the right diaphragm (Fig. 2C, D). After limited mobilization of the right liver, the caudal paracaval portion was dissected from the inferior vena cava (IVC). Careful dissection between the paracaval portion and the IVC continued to make a tunnel, in which a Penrose drain was inserted for liver hanging (Fig. 3A).

The proximal hepatoduodenal ligament was dissected to isolate the right hepatic artery (RHA) and right portal vein (RPV) (Fig. 3B). A hemihepatic block of the RHA and RPV induced hemihepatic discoloration at the liver surface, which was marked with electrocautery. The hepatic parenchyma was transected with Cavitron ultrasonic aspirator and electrocautery without inflow occlusion. The segment $\mathrm{V}$ hepatic vein (V5) branch was transected from the middle hepatic vein (MHV) trunk (Fig. 3C). Under the application of liver hanging, the paracaval portion was elevated to facilitate parenchymal transection.

After near-completion of the hepatic parenchymal transection, two radio-opaque markers were attached at the transection site of the right hepatic duct (RHD) (Fig. 3D), and a second cholangiogram was taken to confirm the transection site at the RHD. The RHD was sharply transected and then the remaining paracaval portion was finally transected (Fig. 4A). The RHD stump was meticulously repaired and a leak test was performed with indigo carmine solution. The operation time from skin incision to repair of the RHD stump took approximately 100 minutes.

After the recipient-side operation was ready to perform total hepatectomy, right liver graft harvest was initiated with a bolus injection of heparin 5,000 units. Routine tran-
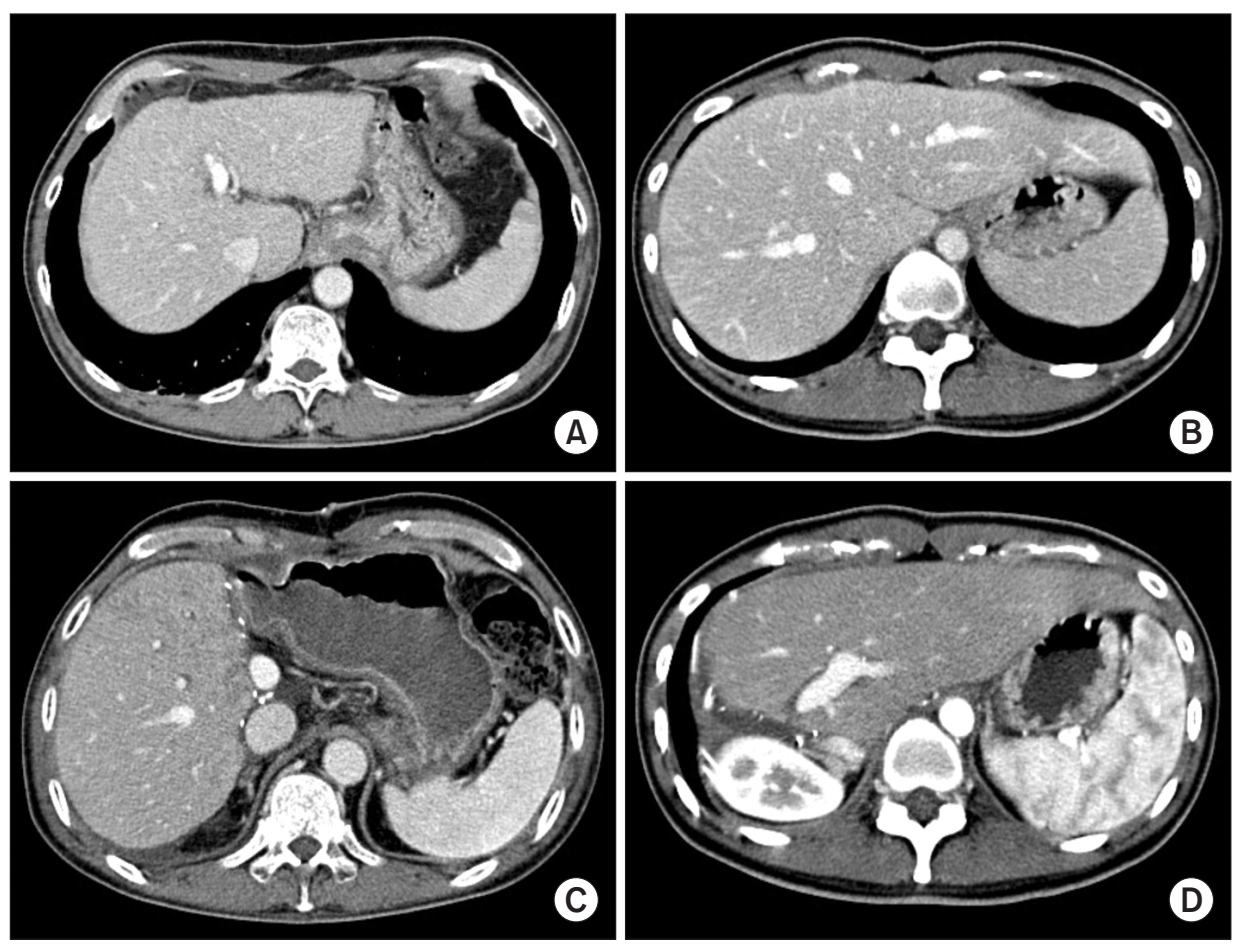

Fig. 1. Peritransplant computed tomography (CT) images. Pretransplant CT images of the recipient (A) and donor (B). Posttransplant CT images of the recipient (C) and donor (D) taken one week after transplantation. 

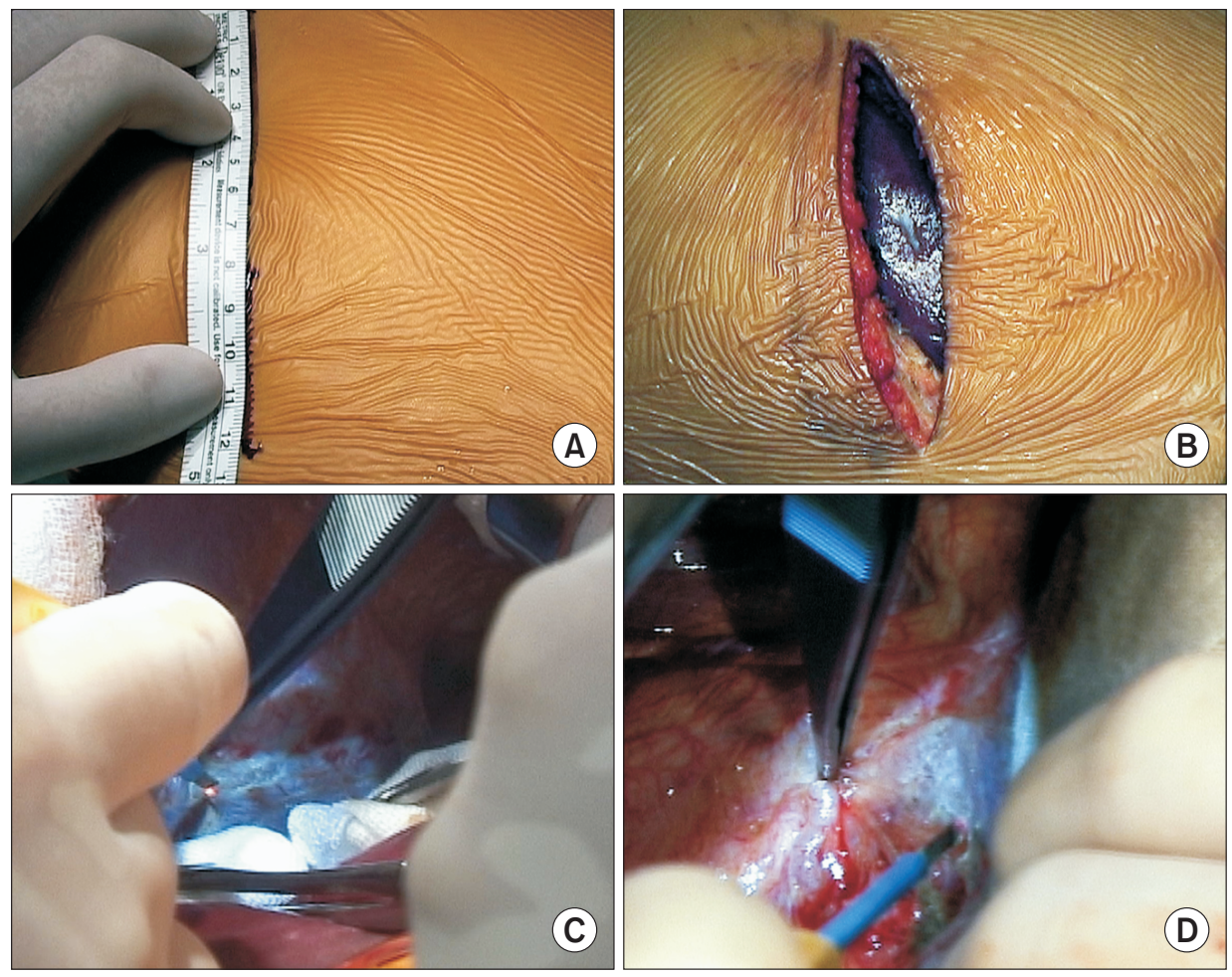

(D)
Fig. 2. Intraoperative photographs of donor right hepatectomy through minimal-incision laparotomy. (A, B) A 12-cm-long subcostal incision is made. (C, D) The liver is carefully mobilized from the right diaphragm.
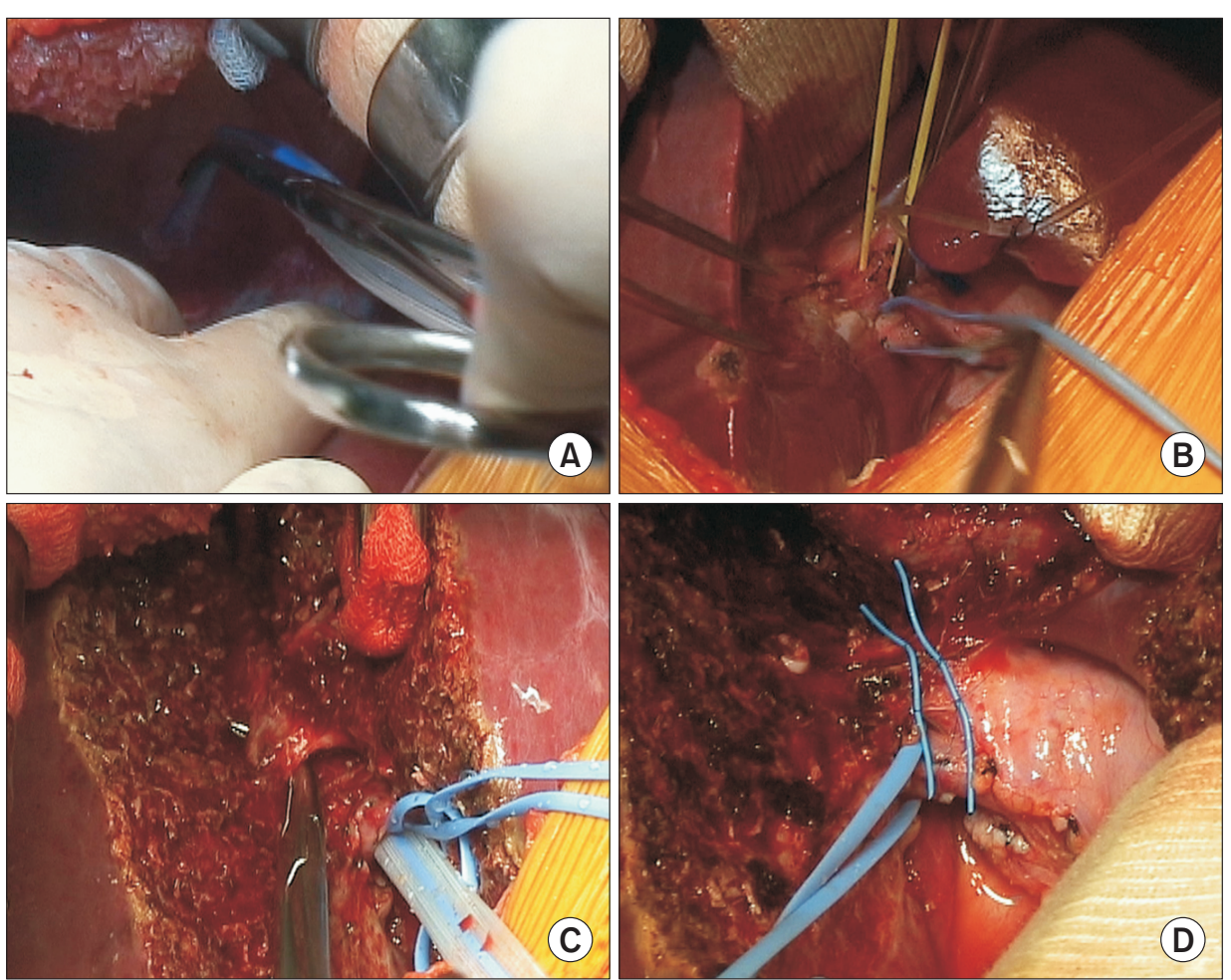

Fig. 3. Intraoperative photographs of donor right hepatectomy through minimal-incision laparotomy. (A) A Penrose drain is inserted beneath the right liver for hanging. (B) The right hepatic artery and portal vein are dissected and encircled with vessel loops. (C) The segment $V$ hepatic vein is transected from the middle hepatic vein trunk. (D) Two radio-opaque markers are attached at the transection site of the right hepatic duct. 
section of the RHA and RPV was followed (Fig. 4B). There were two medium-sized inferior hepatic veins, that were transected together with deep side clamping of the IVC. The right hepatic vein was transected and the right liver was pulled out through the skin incision (Fig. 4C). The RHV and inferior RHV stumps were repaired (Fig. 4D). A third cholangiogram was taken to confirm the absence of donor bile duct stenosis. The skin wound was repaired with interrupted subcutaneous sutures (Fig. 5).

The graft weighed $780 \mathrm{~g}$, resulting in a graft-to-recipient weight ratio of 1.32. Patch venoplasties were applied to the $\mathrm{RHV}$ and inferior RHV to facilitate reconstruction to the recipient IVC. A cryopreserved iliac vein conduit was attached to
V5 for MHV reconstruction. This modified right graft was implanted according to standard procedures of LDLT. The donor recovered uneventfully an was discharged at 9 days after donation (Fig. 1D). The recipient also recovered uneventfully and was discharged at day 17 after transplantation (Fig. 1C), and has been doing well for 12 years after LDLT.

\section{DISCUSSION}

The LDLT operation in the present study was performed in 2009, based on our early experience of MIL. Hundreds of MIL cases have been performed in our institution during the last 12 years, and some technical modifications, includ-
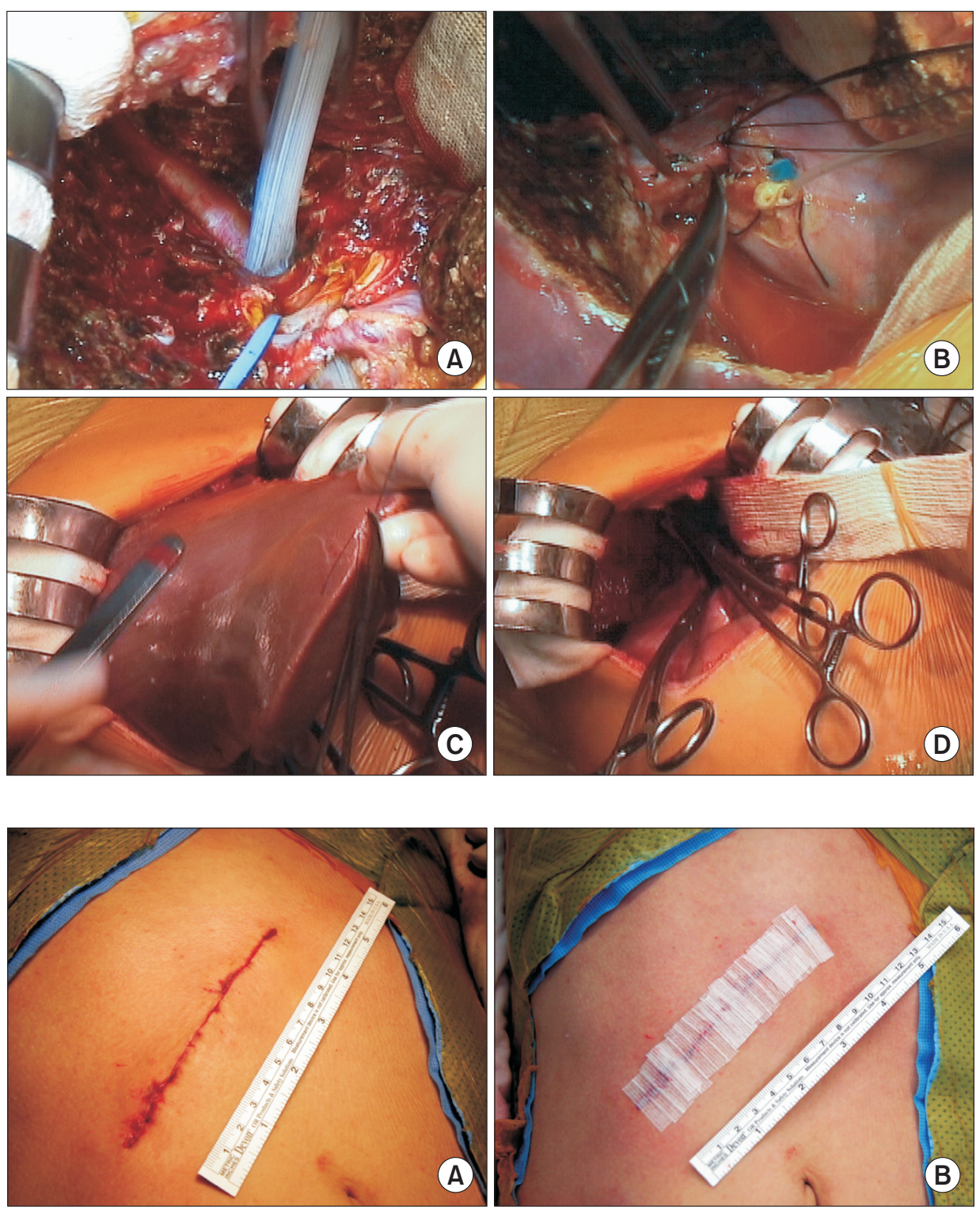

Fig. 4. Intraoperative photographs of donor right hepatectomy through minimal-incision laparotomy. (A) The right hepatic duct is sharply transected and then the remaining paracaval portion is transected. (B) Transection of the right hepatic artery and portal vein is done. (C) The right liver graft is pulled out through the skin incision. (D) The right hepatic vein and inferior right hepatic vein stumps are repaired.

Fig. 5. Intraoperative photographs of minimal-incision laparotomy. The skin wound is repaired with interrupted subcutaneous sutures (A), and sterile strips are attached (B). 
ing the frequent use of vascular staplers during RHV transection, were added to enhance technical convenience and safety of the surgical procedures.

While living donor hepatectomy with MIL enhances the cosmetic aspect of the abdominal skin incision, it is a demanding procedure for donor surgeons because the operative view for right liver mobilization is poor. Accidental bleeding from the IVC or right adrenal gland should be avoided because bleeding control at these sites is difficult because of the poor operative fields. In practice, it is unnecessary to resect the IVC wall excessively because the RHV and inferior RHV stumps of a liver graft can be widened through customized patch venoplasty [9].

The size of the minimal incision that is required for right liver donation is the incision length enabling secure right liver graft delivery. We previously reported that at least a $10 \mathrm{~cm}$-long incision is necessary for delivery of a smallsized right liver graft of approximately $500 \mathrm{~g}$, but a 700 g-weighing graft requires a slightly larger incision of $12 \mathrm{~cm}$ in length. Currently, a skin incision of $10-12 \mathrm{~cm}$ is regarded as MIL $[1,2]$. However, a smaller incision is not always beneficial to the donors because excessive tension at the skin incision edges can worsen the cosmetics of the skin incision [10].

There are various approaches for MIL. The experience of an upper midline incision was reported a long time before, but this incision is placed vertically against the abdominal skin crease, leading to the formation of an easily recognizable scar [4]. We have also performed minimal upper midline skin incision, but the indication is usually confined to the left liver harvest. For the right liver harvest, we have preferentially used a right subcostal incision (Fig. 6). For video-assisted MIL, the upper midline, right transverse, or right subcostal incision was used according to the institutional preferences $[1,5,7,11]$.

We previously presented our experience for right liver graft harvest with HALS [1], in which we found that HALS facilitated dissection of the retrohepatic IVC, therefore preventing excessive liver retraction during right liver mobilization and IVC dissection. However, the application of video equipment appears to be inconvenient for the surgeons. Recently, total laparoscopic hepatectomy has been increasingly performed in many Korean LDLT centers, including our institution $[3,12]$. Although satisfactory outcomes of LDLT combined with total laparoscopic donor hepatectomy have been reported, it is still regarded as a challenging procedure requiring strict selection of living donors [12,13]. We have worried about donor safety as well as biliary complication of the recipients in cases of total laparoscopic hepatectomy $[13,14]$.
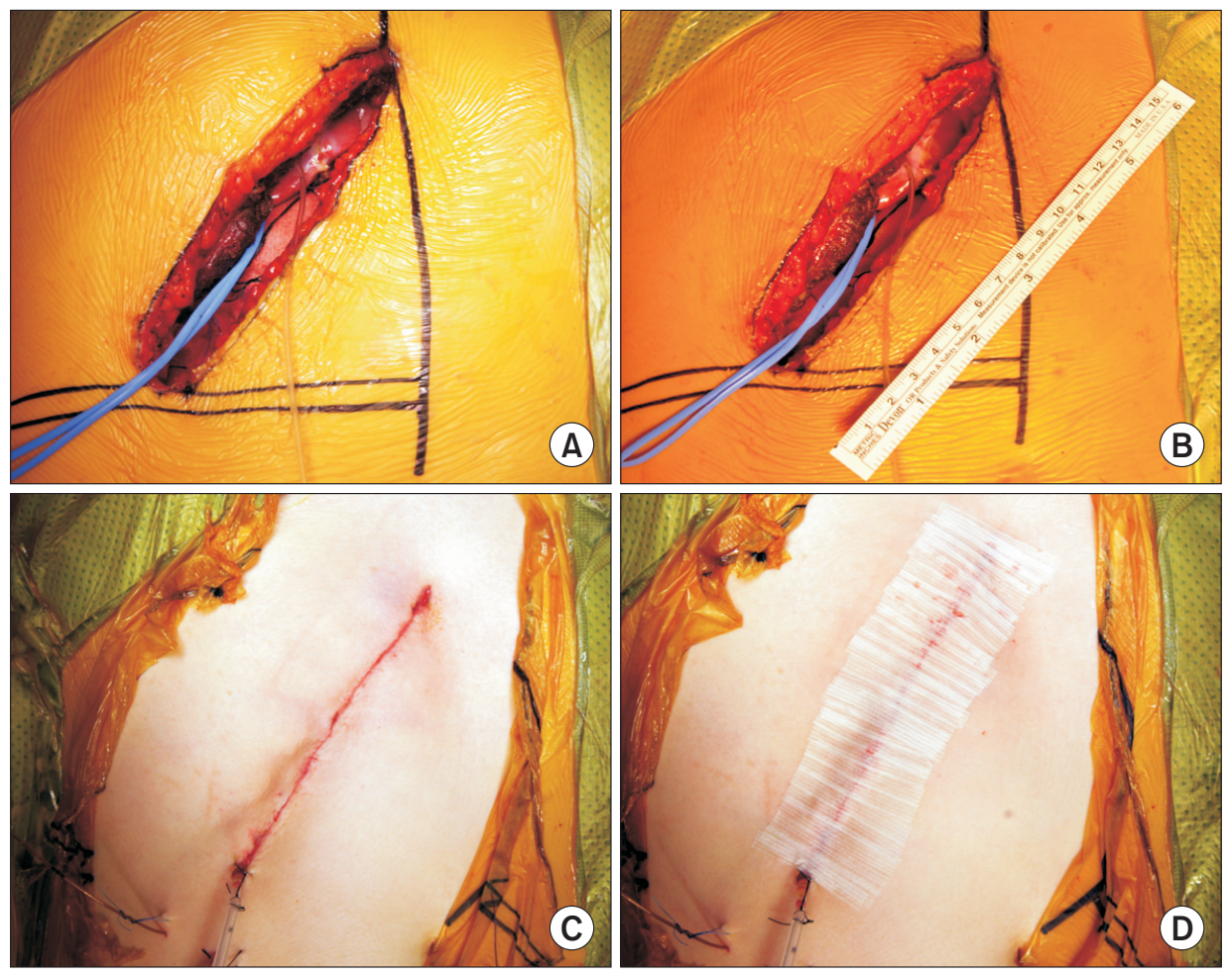

Fig. 6. Photographs of a right subcostal skin incision applied to harvest of a right liver harvest. (A, B) A right subcostal skin incision is made corresponding to the hypotenuse of a triangle drawn at the abdomen. (C, D) The skin wound is repaired with interrupted subcutaneous sutures with an underlying drain, and sterile strips are attached. 
We have progressively widened the indication of MIL, but it is often limited in living donors with high body mass index because such donors usually require a wider operative view for secure liver mobilization. Parenchymal transection per se with liver hanging maneuver is a kind of anterior approach, thus it does not require a wide operative view [2].

Based on our experience, we believe that MIL is a compromise option between the conventional skin incision and total laparoscopic hepatectomy regarding cosmetics of the skin incision and donor safety, although it is a demanding procedure for donor surgeons.

\section{SUPPLEMENTARY MATERIAL}

Supplementary data related to this article can be found online at https://doi.org/10.52604/alt.21.0023.

\section{FUNDING}

There was no funding related to this study.

\section{CONFLICT OF INTEREST}

All authors have no conflicts of interest to declare.

\section{ORCID}

Tae-Yong Ha https://orcid.org/0000-0001-9932-0212

Dong-Hwan Jung https://orcid.org/0000-0001-5984-023X

Gi-Won Song https://orcid.org/0000-0002-4235-0434

Gil-Chun Park https://orcid.org/0000-0003-1631-3258

Shin Hwang https://orcid.org/0000-0002-9045-2531

\section{AUTHORS' CONTRIBUTIONS}

Conceptualization: TYH, SH. Data curation: TYH, DHJ, GWS, GWS, GCP. Formal analysis: TYH. DHJ. Investigation: All. Methodology: All. Project administration: SH. Validation: SH. Visualization: $\mathrm{SH}$. Writing - original draft: $\mathrm{TYH}, \mathrm{SH}$. Writing - review \& editing: TYH, DHJ, GWS, SH.

\section{REFERENCES}

1. Ha TY, Hwang S, Ahn CS, Kim KH, Moon DB, Song GW, et al. Role of hand-assisted laparoscopic surgery in living-donor right liver harvest. Transplant Proc 2013;45:2997-2999.

2. Nagai S, Brown L, Yoshida A, Kim D, Kazimi M, Abouljoud MS. Mini-incision right hepatic lobectomy with or without laparo- scopic assistance for living donor hepatectomy. Liver Transpl 2012;18:1188-1197.

3. Hong SK, Choi GS, Han J, Cho HD, Kim JM, Han YS, et al. Pure laparoscopic donor hepatectomy: a multicenter experience. Liver Transpl 2021;27:67-76.

4. Shen S, Zhang W, Jiang L, Yan L, Yang J. Comparison of upper midline incision with and without laparoscopic assistance for living-donor right hepatectomy. Transplant Proc 2016;48: 2726-2731.

5. Eguchi S, Takatsuki M, Soyama A, Hidaka M, Tomonaga T, Muraoka I, et al. Elective living donor liver transplantation by hybrid hand-assisted laparoscopic surgery and short upper midline laparotomy. Surgery 2011;150:1002-1005.

6. Suh SW, Lee KW, Lee JM, Choi Y, Yi NJ, Suh KS. Clinical outcomes of and patient satisfaction with different incision methods for donor hepatectomy in living donor liver transplantation. Liver Transpl 2015;21:72-78.

7. Kurosaki I, Yamamoto S, Kitami C, Yokoyama N, Nakatsuka H, Kobayashi T, et al. Video-assisted living donor hemihepatectomy through a 12-cm incision for adult-to-adult liver transplantation. Surgery 2006;139:695-703.

8. Koffron AJ, Kung R, Baker T, Fryer J, Clark L, Abecassis M. Laparoscopic-assisted right lobe donor hepatectomy. Am J Transplant 2006;6:2522-2525.

9. Jung DH, Hwang S, Ahn CS, Moon DB, Ha TY, Song GW, et al. Quilt unification venoplasty of the right hepatic veins enabling double inferior vena cava anastomosis in living donor liver transplantation using a right liver graft. Ann Liver Transplant 2021;1:86-94.

10. Khansa I, Harrison B, Janis JE. Evidence-based scar management: how to improve results with technique and technology. Plast Reconstr Surg 2016;138(3 Suppl):165S-178S.

11. Nitta H, Sasaki A, Fujita T, Itabashi H, Hoshikawa K, Takahara T, et al. Laparoscopy-assisted major liver resections employing a hanging technique: the original procedure. Ann Surg 2010; 251:450-453.

12. Rhu J, Kim KD, Choi GS, Kim JM, Kim GS, Joh JW. Decreasing the operation time of living donor liver transplantation in the era of laparoscopic living donor hepatectomy. Ann Liver Transplant 2021;1:24-28.

13. Zhang W, Xu L, Zhang J, Che X. Safety and feasibility of laparoscopic living donor right hepatectomy for adult liver transplantation: a meta-analysis. HPB (Oxford) 2021;23:344-358.

14. Jeong JS, Wi W, Chung YJ, Kim JM, Choi GS, Kwon CHD, et al. Comparison of perioperative outcomes between pure laparoscopic surgery and open right hepatectomy in living donor hepatectomy: propensity score matching analysis. Sci Rep 2020;10:5314. 